\title{
Research Progress of Bone Tumor Clinical Diagnosis
}

\author{
Rui Jiang ${ }^{1}$, Ziyan Zhang ${ }^{2}$, Han $\mathrm{Wu}^{1}$ and Chao Zhang ${ }^{3, \text { a, * }}$ \\ ${ }^{1}$ Department of orthopedics, China-Japan Union Hospital of Jilin University, Jilin 130033, China \\ ${ }^{2}$ Department of orthopedics, The Second Hospital of Jilin University, Jilin 130041, China \\ ${ }^{3}$ Department of ophthalmology, The Second Hospital of Jilin University, Jilin 130041, China \\ azhangchao0701@163.com
}

Keywords: bone tumor, clinical features, diagnostic method.

\begin{abstract}
Bone tumor belongs to a tumor among human skeletons or skeleton affiliated groups. It is the result of cytogenetic material abnormal expression, which is same as other tumors. Bone tumor belongs to a common disease. In recent decades, research on bone tumor clinical diagnosis is rapidly developed, which becomes a basic and key discipline for studying bone tumors. Bone tumor pathological classification is gradually determined with gradual maturity of molecule detection technique. Bone tumors are divided into osteogenic tumor, chondrogenesis tumor, giant-cell tumor of bone, bone marrow neoplasms, vascular tumor, etc. Its occurrence, development and metastasis rules thereof are gradually revealed from the molecular perspective through studying clinical diagnosis of bone tumors. The paper has important significance for disease prevention, treatment and post-operative postoperative rehabilitation.
\end{abstract}

\section{Introduction}

Bone tumor clinical diagnosis has become an important branch in diagnostics with the increasing prominence of clinical diagnosis in modern medical diagnosis. Because bone tumor incidence is low, bone tumor patients accepted by each pathologist are very limited even in large-scale general hospitals each year, it is difficult to use long-term working experience to judge their etiology and establish treatment plans. Therefore, we need to analyze it from the aspects of clinical data, imaging features, molecular biology, microscopic observation, etc. in order to accurately diagnose bone tumor patients, and finally the patients are diagnosed. In the paper, some basic operations and skills in bone tumor clinical diagnosis are mainly introduced. The diagnosis direction beneficial for most bone tumors are accurately judged through repeated practice.

\section{Clinical features}

Detailed clinical data is the first step in bone tumor diagnosis. It generally includes the following aspects: age, gender, lesion site, signs and symptoms; disese causes, disease duration, personal disease history, family disease history, trauma history, treatment history, systemic diseases and systematic physical examination. The patient age and disease site are the most valuable clinical data for bone tumor diagnosis [1].

Age features: there is certain disease high-incidence season aiming at most bone tumors, the high-incidence seasons are different for benign and malignant tumors as well as neoplasia, and they can be discovered from 10 to 49 years old. People from 1 to 4 years old are prone to metastatic neuroblastoma, and it belongs to a malignant tumor, which is derived from adrenal gland. Metastatic neuroblastoma is a common tumor for newborns. The pathogenesis is not clear at present. Age from 10 to 19 years old is the high-incidence season of bone tumors, and such tumors mostly belong to benign tumor and rarely belong to malignant tumors [12]. Benign tumors include solitary bone cyst, which is derived from aneurysmal bone cyst, chondroblastoma, etc. Malignant tumors include osteosarcoma, Ewing's sarcoma, etc. Wherein, scientific analysis shows that Ewing's sarcoma is derived from neuroderm, the form belongs to indifferent globular cell. People from 10 to 29 years old 
are prone to benign tumors of osteoblastoma and chondromyxoid fibroma. People from 20 to 39 years old are prone to malignant giant-cell tumor of bone, and its form is bleeding granulation tissue according to naked eye observation, which is related to trauma. People from 20 to 49 years old are prone to hemangioma and enchondroma, wherein enchondroma is also called mesenchymal chondroma, lesion removal and autogenous bone graft operative treatment can be adopted with excellent treatment effect. People from 30 to 50 years old are prone to fibrosarcoma, ameloblastoma and chordoma. People from 40 to 69 years old have weaker constitution with age, and the morbidity of myeloma and lymphoma is greatly increased.

Pathogenic site features: relationship of age and pathogenic site: the incidence of some bone tumors is high at specific age. For example, $80 \%$ solitary bone cysts are discovered in femur and humerus of patients younger than 17 years old, $52 \%$ solitary bone cysts are discovered in pelvis and STA of patients older than 17 years old. In addition, isolated eosinophilic granuloma is mostly discovered in femur, vertebra, skull, pelvis and other sites of patients younger than 20 years old, and in rib, mandible and other sites of patients from 20 to 30 years old, and the disease is rare for people older than 30 years old. Relationship of bone category and pathogenic site: most tumors and tumor-like lesions are located in long bones; flat bone and irregular bone are more prone to osteoblastoma, Ewing's sarcoma, hemangioma, etc. Aneurysmal bone cyst not only is discovered in flat bone and irregular bone, but also is available in long bone. It is mostly observed in four limb long bone and vertebral lamina of teenagers; chondroma is generally discovered in short bone. In one STA, most tumors are concentrated in metaphysis, such as non-ossifying fibroma, parosteal osteosarcoma, periosteal chondrosarcoma, etc.; chondroblastoma is commonly discovered in epiphysis; osteoblastoma and osteoidosteoma are commonly discovered in backbone. Predilection site of benign bone tumor and tumor-like lesio: skull and facial bone are frequently prone to osteoblastoma, Langerhans cell histiocytosis, etc. in axial skeleton; jaws are frequently prone to giant cell reparatuve granuloma, ossifying fibroma, etc.; vertebra is frequently prone to hemangioma, aneurysmal bone cyst, etc. Four-limb long bones are frequently prone to simple bone cyst, chondromyxoid fibroma, intraosseous ganglion, etc. Hand and foot bones are frequently prone to woven reactive periostitis, giant cell reparatuve granuloma, etc. Malignant bone tumor predilection site: skull and facial bone are more prone to metastatic neuroblastoma, multiple myeloma, etc. in axial skeleton. Vertebra is frequently prone to metastatic carcinoma, chordoma, etc; rib sternum is more prone to chondrosarcoma. Long bones are more prone to malignant fibrous histiocytoma, chondrosarcoma, etc. Hand and foot bones are rarely prone to malignant tumor.

Relationship of gender and bone tumor: male people are more prone to osteoid sarcoma and myeloma; female people are mostly prone to hemangioma and giant-cell tumor. It is closely related to female and male body structures as well as environment factors in many aspects.

\section{Imaging diagnosis}

Imaging diagnosis has important medical significance to judge bone tumor benign malignant types and bone tumor histological structure. In various imaging diagnosis forms, plain film is the most effective to identify bone tumors. Bone formation and bone destruction can be displayed in the plain film on the one hand, many pathological changes of periosteum and bone surrounding tissues also can be identified on the other hand [9] . X-ray plain film is still the most effective and most concise mode for examining bone tumors [10]. When X-ray penetrates through bone, bone, surrounding skin tissues and cartilage tissues have prominent difference to X-ray absorption, nature black-white images with different densities and distinct gradations are formed on the film. Great convenience is provided for diagnosis of bone tumors. Though CT, MRI and other modern imaging means make great progress, $\mathrm{X}$ -ray is still the primary routine examination mode in bone tumor diagnosis [11]. After X-ray plain film is produced, the film should be analyzed and diagnosed according to the following steps: observe the position of bone tumor in the whole body skeletons; observe the morphological structure of bone tumor; observe the density of bone tumor in the lesion area; observe whether the lesion belongs to 
single or multiple lesion; observe the form chance of tissues and organs around the bone tumor. The changes of bone tissues and even osteoma categories are analyzed as a whole through above observation. The final conclusion can be obtained through combination with clinical detection and molecular biology analysis, thereby determining whether it is necessary to further use more precise imaging technology for inspection or not. In the X-ray plain film inspection, analysis on the bone density, cortical area corrosion degree, expansion degree and penetration role is particularly important. Meanwhile, periosteum changes thereof also should be observed, such as change under solar radiation, etc.

The similarity and difference of bone tumor and normal bone or other tissues in X-ray plain film are mainly manifested on increase and decrease of density thereof. Density change is determined by osteoblast and osteoclast [13]. The increase or decrease of bone density will result in different visual forms of X-ray plain films [14]. For example, X - ray plain film shows swelling bone destruction damage, the damage is generally located in the center of the medullary cavity aiming at bone cyst in benign bone tumor. The cyst cavity contains brown transparent liquid or blood color liquid, its composition components are the same as serum. The outermost layer is covered by a very thin film-shaped hardening layer. The density in the cyst is even aiming at bone cyst patients. However, surrounding bone fibers show abnormal proliferation status, and they have different forms and density reduction, which can be clearly observed in the X-ray plain film. In addition, small round cell sarcoma (Ewing sarcoma) also belongs to a typical example with bone mineral density decrease [15]. Normal bone tissues show small-area bone destruction with wireless value increase of tumor cells and surrounding tissue hyperplasia in the early stage of tumor occurrence. It is gradually moved to the direction of normal bone. Small round cell sarcoma gradually invades to the bone cortical area and some periosteum is opened as the disease progresses gradually. The constant destruction of the new bone tissue will result in onion skin periosteum reaction with the proliferation of cancer cells. Middle stage is started next, the tumor will break through the new bone and form a larger soft tissue mass with disease focus diffusion. If there is a pathological fracture, it is possible that the bone tumor appears in the high stress zone.

It is generally associated with new bone and cartilage tissue calcification aiming at bone density increase. In diagnosis, If new bone density increase or cartilage tissue calcification is discovered in the X-ray plain film by a doctor during diagnosis, the bone tumor patients can be basically diagnosed. There are also several typical cases of bone density increase, such as neoplastic bone, neoplastic cartilage calcification, reactive new bone, etc. Osteoidosteoma is a typical case of neoplastic bone. It is displayed as a round area with small diameter in X-ray plain film, and it is wrapped by an even hardened shell-shaped regional tissues. The above form is the most typical existence form of osteoidosteoma. The osteoidosteoma form in hands, feet, limb bones and vertebral part can be slightly different from the above contents. The osteoidosteoma in different sites of periosteum, medullary space and cortex have different symbols in X-ray plain film, such as tumor-like lesion on spine. Since spine has more complicated organization structure, it can be easily shaded by various other soft tissues in the surrounding area on the X-ray plain film. The film is produced from many perspectives. The films in different stages have greater difference clinically. Accurate diagnosis of tumor is greatly difficult. However, since the tumor grows on the spine, the patients suffer from radioactive neuropathic pain during spine bending at the same time. It is also called painful scoliosis. When the phenomena is available, suspected osteoidosteoma is basically diagnosed. However, it is not necessarily caused by osteoidosteoma, and it is also necessary to carry out many other auxiliary inspections.

\section{Molecular biology inspection}

The inspection at bone tumor cellular level and molecular level is also gradually perfected with the development of medical cell biology and biochemistry. The commonly used inspection methods include flow cytometry analysis technique, in-situ molecular hybridization technique, etc. [8]. 
Flow cytometry: flow cytometry refers that laser beams are used for illuminating small particles floating in the fluid, thereby it can generate electromagnetic movement. Specific tiny particles are sorted, and flow cytometry has counting function. The technique can be used for continuous parameter analysis on some or a variety of similar cells. It is a high-speed, efficient and convenient biological analysis technique.[2]. It is combined with optics, fluid mechanics, electromagnetism and other disciplines. It belongs to both cell sorting technology and cell analysis technology. The formation of bone tumor is a process from qualitative change of cell proliferation to deterioration and genetic material change. The role of flow cytometry in the process of tumor diagnosis cannot be ignored. If DNA aneuploid cell peak is found during the inspection process, it can provide a strong basis for the diagnosis of bone tumor [3]. Currently, single flow cytometry cannot be used as the sole basis for accurate diagnosis of tumor. It also should be combined with other modes such as clinical inspection, pathology inspection, etc., thereby getting accurate conclusions.

In-situ molecular hybridization technique: each nucleic acid contains mutually-complementary basic-group chain, which follows the principle of basic-group complementary pairing. The radioactive DNA or RNA probe is injected into cells, the probe will be combined with complementary specific nucleic acid molecule chain thereof, thereby forming a specific nucleic acid molecular complex. The tumor is inspected through certain inspection method. It is a basic inspection method on malignant tumor from the gene perspective [4]. In-situ molecular hybridization techniques have been widely used at present stage. It belongs to the most accurate and most effective pathology inspection means in the aspect of malignant tumor inspection. It can be qualitatively and quantitatively analyzed from the gene level [5].

Microscope observation: microscope observation is divided into microstructure observation and submicroscopic structure observation. In the past, people often used ordinary microscope for bone tumor morphology observation, staining identification, etc. due to the limitations of science and technology. Microscopic observation of tumor gradually tends to the submicroscopic structure observation with the invention of the electron microscope. Its disease causes, initial position, pathogenesis, etc. are judged according to its form and growth features under electron microscope [6].

According to the above-introduced comprehensive crossed diagnosis in four aspects of clinical data, imaging diagnosis, molecular biology inspection and microscopy observation, accurate diagnosis can be obtained [7]. The inspection method of bone tumor is becoming more and more perfect because of the improvement of laboratory infrastructure and doctor professional level. It is of great significance to the study on tumor occurrence mechanism, metastasis direction and disease metastasis.

\section{Acknowledgments}

This work was supported by The study of c-Myc and mTOR inhibitors in the combined targeting therapy of osteosarcoma. Jilin provincial finance department (No 3D517P373430).

\section{References}

[1]. Yang Qingcheng, Mei Jiong. Clinical treatment progress of malignant bone tumor .Foreign Medical Orthopedics Branch,2001,22 (1):32-33.

[2]. Guo Wei, Yang Rongli, Tang Xiaodong, etc. Analysis on treatment effect of osteosarcoma new adjuvant chemotherapy drug treatment Chinese Medical Journal, 2004,84 (7):1187.

[3]. Guo Wei, Yan Taiqiang. Chemotherapy progress of malignant bone tumor Chinese Journal of Oncology ,2002,24 ( 5 ):5 17 .

[4]. Wittig JC,Bickels J,Priebat D,et al.Osteosarcoma:a multidisciplinaryapproach to diagnosis and treatmen Am Fam Physician,2002,65:1123 1132. 
[5]. Paulussen M, Frohlich B, Jurgens H.Ewing tumour:incidence, prog-nosis andtreatment options paediatr Drugs,2001,3:899 913.

[6]. Uchida A,Myoui A,Araki N,et al.Neoadjuvant chemotherapy forpediatric osteosarcoma patients Cancer, 1997,79:411 415.

[7]. Cai Youbo, Niu Xiaohui, Zhang Qing, etc. Long-term outcome of limb primary osteosarcoma comprehensive treatment. Chinese Journal of Surgery, 2000,38:329-331.

[8]. Ma Zhongtai, Li Huaiguang, Shi Xuedong. Osteosarcoma chemotherapy and treatment effect judgment. Chinese Journal of Orthopaedics, 2000,20 (supplementary issue):44-46.

[9]. Tsuchiya H, Tomita k, Mori Y,et al.Marginal excision for os-teosarcoma with caffeine assisted chemotherapy.Clin orthop,1999,358:27 35.

[10].Weis LD.Adolesc Med.1999,10 (3):451 453.

[11].Feng Chuanhan. Progress of bone tumor diagnosis and treatment. Chinese Journal of Orthopaedics ,2000,20 (2)supplementary issue:10.

[12].Aberg M,Rydholm A,Holmberg J,et al.Reconstruction with a free Vascularized fibular.graft for malignant bone tumor.Acta Orthop scand.1988,59:430 437.

[13].Finkemeier CG Bone-grafting and bone-graft substitutes J BoneJoint Surg (Am), 2002, 84 (3):454 464.

[14].Edited by Guo Wei [translator]. Diagnosis and treatment of bone and soft tissue tumor. Tianjin: Tianjin Technology Translation Translation Co., Ltd., 2007,1:154.

[15].Delaney TF, Trofimov AV,Engelsman M,et al.Adancedtechnologyradiation therapy in the management of bone and soft tissue sarcomas.Cancer Control,2005,12:27 35. 